\title{
L'instrumentation scientifique sur sources synchrotrons : principes et concepts généraux
}

Jean Susini (susini@esrf.fr)

ESRF, BP 220, 38043 Grenoble Cedex

L'émergence de nouvelles

sources synchrotrons et

l'exploitation de faisceaux

de rayons $\mathrm{X}$ aux propriétés

exceptionnelles ont appelé

le développement d'une

instrumentation scientifique

de plus en plus sophistiquée.

Les interactions des rayons $X$

avec la matière sont

particulières et définissent

par nature le champ des

possibles dans la conception

des optiques et des détecteurs

de rayons $X$, et de l'environ-

nement des échantillons.

En partant d'une perspective

historique, cet article présente

une introduction générale des

grands principes sur lesquels

I'instrument synchrotron

repose aujourd'hui.
Dès la découverte des rayons $\mathrm{X}$ par Röntgen, la possibilité de les utiliser à des fins scientifiques a motivé de nombreux essais de développements instrumentaux. Plusieurs sont restés infructueux et Röntgen lui-même, dans son article original de 1896, avait étonnamment identifié certains des verrous technologiques à lever. Il mentionnait, par exemple :

"l'indice de réfraction [...] ne peut pas être supérieur à 1,05 [...] et en conséquence des lentilles ne peuvent pas concentrer les rayons $X[. .]$.

"les plaques photographiques et les films sont sensibles aux rayons $X$ et offrent un moyen intéressant d'enregistrer leurs effets [...]"

"la détection de phénomènes d'interférences a été essayée, mais sans succès, probablement à cause de leur faible intensité [...]".

Il est remarquable que Röntgen ait immédiatement et de façon purement empirique réussi à délimiter les enjeux du développement de l'instrumentation pour rayons $\mathrm{X}$. En bref, la nature très particulière de leur interaction avec la matière a singulièrement compliqué la tâche, et la réalisation d'optiques et de détecteurs pour rayons $\mathrm{X}$ reste donc, même de nos jours, un défi technologique. De même, il a fallu attendre les années 1970 et l'émergence de sources $\mathrm{X}$ très lumineuses, les sources synchrotrons, pour motiver et accélérer ces développements instrumentaux.

La communauté scientifique bénéficie aujourd'hui de sources dites de troisième génération, qui offrent une grande brillance (10 ordres de grandeur de plus qu'un tube à rayons $\mathrm{X}$ classique), une large gamme de longueurs d'onde, un contrôle de la polarisation ainsi que de la cohérence du faisceau. De nombreux développements instrumentaux ont été nécessaires pour une exploitation optimale des possibilités offertes par ces nouvelles sources. Par nature, chaque discipline scientifique utilisant le rayonnement synchrotron a développé une instrumentation qui lui est propre, et il est impossible en quelques pages d'en exposer l'étendue. Cet article se limitera donc à une introduction générale sur les grands principes et sur les développements les plus marquants ou les plus répandus.

\section{Un court rappel \\ sur les interactions rayonnement-matière}

Les méthodes d'analyse X et leurs instrumentations associées sont principalement déterminées par les interactions des rayons $\mathrm{X}$ avec la matière de l'échantillon. Dans ce contexte, il est important de rappeler les différents domaines usuels du spectre électromagnétique couvrant la gamme des rayons X. En fait, les définitions varient dans la littérature et se fondent traditionnellement sur les modes de production du rayonnement, son pouvoir de pénétration et le type de monochromateur utilisé. Pour la suite, nous retiendrons les conventions suivantes ${ }^{(a)}$ : - rayons X mous : $100 \mathrm{eV}$ - $2000 \mathrm{eV}$, soit l'intervalle de longueurs d'onde 124,00 $\AA-6,20 \AA$;

- rayons X tendres : $2000 \mathrm{eV}-8000 \mathrm{eV}$, soit l'intervalle de longueurs d'onde $6,20 \AA-1,55 \AA$;

- rayons X durs : $8 \mathrm{keV}$ - $500 \mathrm{keV}$, soit l'intervalle de longueurs d'onde $1,55 \AA-0,02 \AA$. 


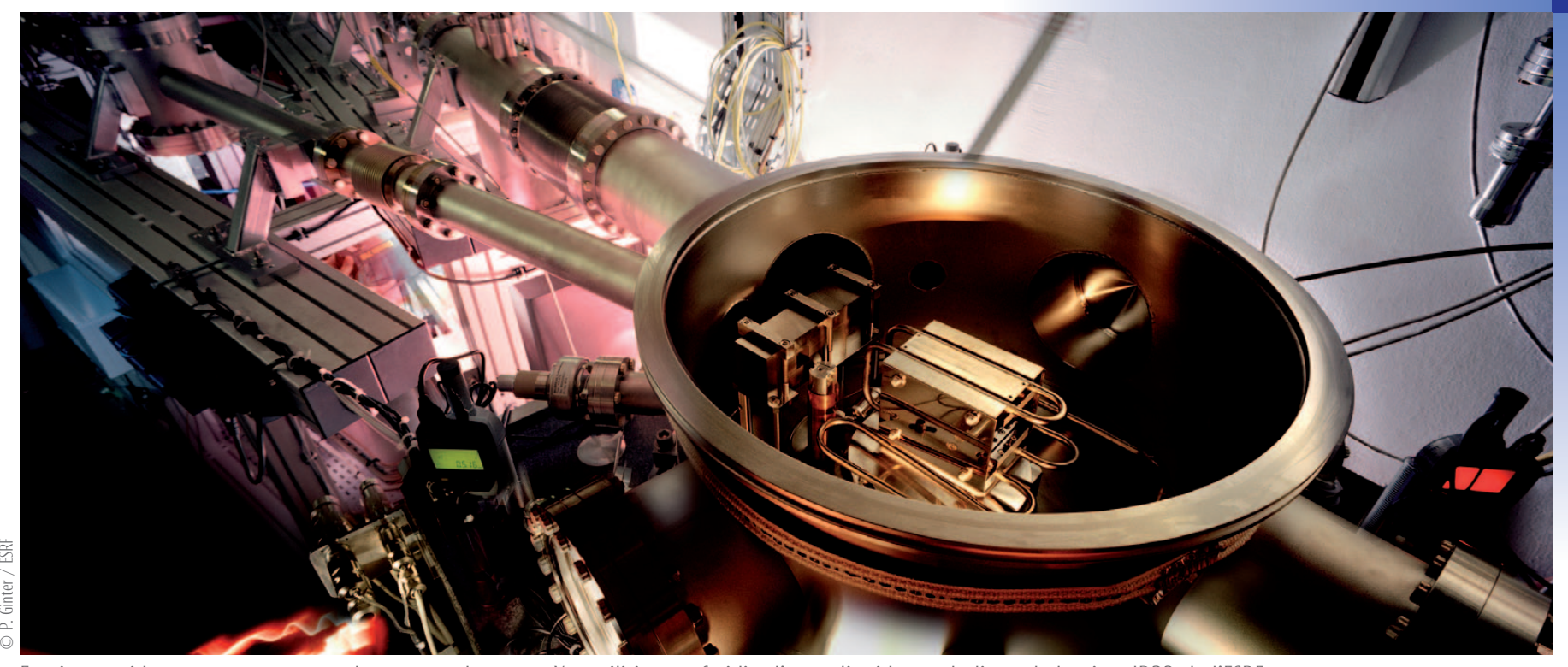

Enceinte à vide contenant un monochromateur de rayons X en silicium, refroidi à l'azote liquide, sur la ligne de lumière ID23 de l'ESRF.

Toute interaction d'un rayonnement électromagnétique avec la matière peut se formuler selon deux descriptions :

- une approche atomique, dans laquelle l'interaction est décrite en termes de perte d'énergie, de diffusion (élastique et inélastique) et d'émissions secondaires (photoémission et fluorescence X) ;

- une approche macroscopique, qui repose sur la définition optique de l'amplitude et de la phase de l'onde transmise en fonction de la densité d'atomes (mais qui ne traite pas les phénomènes de diffusion).

Les deux descriptions sont équivalentes et peuvent être associées dans un formalisme liant les facteurs de diffusion atomique à l'indice optique (ou de réfraction) complexe $n$. Ce dernier est défini par :

$$
n(\lambda)=1-\delta+i \beta
$$

où $1-\delta$ correspond à la réfraction et $\beta$ à l'absorption. Dans la gamme spectrale correspondant aux rayons X durs, l'absorption photoélectrique est l'effet prédominant, en particulier si les photons $\mathrm{X}$ ont une énergie proche de l'énergie de liaison des électrons cibles et également pour des matériaux de numéro atomique élevé. $\beta$ est alors relié à $\mu$, coefficient linéaire d'absorption, et à $\lambda$, longueur d'onde du rayonnement supposé ici monochromatique, par :

$$
\beta=(\lambda / 4 \pi) \mu(\lambda)
$$

Selon la dualité décrite précédemment, les contrastes d'absorption et d'amplitude de l'onde transmise se rapportent à la même technique d'imagerie. Celle-ci est basée sur l'atténuation locale du rayonnement, et le contraste a pour origine la variation d'absorption induite par les différences de densité, d'épaisseur et de composition chimique dans un échantillon hétérogène.
La réfraction peut, de même, être exprimée en fonction de paramètres atomiques par :

$$
\delta=\left(\mathrm{Z}_{V} r_{e} / 2 \pi\right) \lambda^{2}
$$

où $Z_{V}$ est le nombre d'électrons par unité de volume et $r_{e}$ le rayon classique de l'électron $\left(2,8210^{-13} \mathrm{~cm}\right)$.

La plupart des propriétés optiques peuvent être dérivées de l'indice optique. Les composantes réelle $\delta$ et imaginaire $\beta$ de l'indice montrent une dépendance très différente à l'énergie des photons $\mathrm{X}$. En effet, dans le domaine énergétique où l'effet photoélectrique prédomine et loin des seuils d'absorption, en première approximation $\beta \sim E^{-4}$ et $\delta \sim E^{-2}$. Ainsi, pour des rayons $\mathrm{X}$ durs, les valeurs de $\delta$ peuvent être supérieures de plusieurs ordres de grandeurs à celles de $\beta$, offrant des possibilités d'exploiter le phénomène

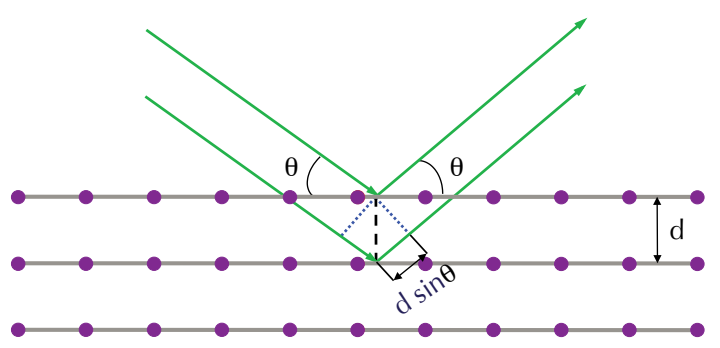

de réfraction tout en limitant les effets d'absorption. De nombreuses techniques d'imagerie de phase, mais aussi la réalisation de nouvelles optiques pour rayons $\mathrm{X}$, découlent de cette particularité.

Par ailleurs, du fait que leurs longueurs d'onde sont de l'ordre de grandeur des distances interatomiques, les rayons $\mathrm{X}$ peuvent être diffractés par toute structure cristalline périodique. La diffraction $\mathrm{X}$ est régie par la loi de $\mathrm{Bragg}^{(\mathrm{b})}$ :

$$
2 d \sin \theta_{\mathrm{B}}=n \lambda
$$

où $d$ est la distance entre deux plans atomiques du cristal, $\lambda$ la longueur d'onde de la lumière incidente, $\theta_{\mathrm{B}}$ l'angle d'incidence du faisceau lumineux et $n$ l'ordre de diffraction $(n=0, \pm 1, \pm 2 \ldots)$ (fig. 1). Ce phénomène de diffraction est à la base de la sélection en énergie par les cristaux monochromateurs.

\〉 a b Géométrie de Bragg

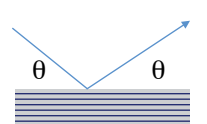

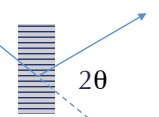

Géométrie de Laue
1. Principe géométrique de la loi de Bragg. (a) Le phénomène de diffraction correspond à la superposition des ondes réfléchies sur les différents plans atomiques successifs. Si $\theta$ est l'angle d'incidence, la différence de marche entre les deux faisceaux (verts) est $2 d \sin \theta$. Un maximum d'intensité est obtenu quand cette différence de marche est un multiple entier de la longueur d'onde : $2 d \sin \theta=n \lambda$. Les deux géométries en réflexion ou en transmission sont dites de Bragg (b) ou de Laue (c). Dans la géométrie de Bragg, les faisceaux incident et réfléchi sont monochromatiques; dans celle de Laue, les faisceaux incident et transmis sont polychromatiques, le faisceau réfléchi est monochromatique. 


\section{\) \\ Les systèmes optiques}

Les expériences utilisant la lumière, quelle que soit la longueur d'onde considérée, nécessitent généralement d'ajuster les propriétés du faisceau aux conditions expérimentales, et plus particulièrement aux caractéristiques de l'échantillon et de son analyse. Plusieurs paramètres sont alors à considérer dans la définition du faisceau, tels que le flux intégré, la largeur spectrale (ou monochromaticité), la divergence et les dimensions spatiales. Plus récemment, le degré de cohérence longitudinale est un nouveau paramètre à prendre en compte pour certaines expériences d'holographie $\mathrm{X}$ et de diffraction X. L'ensemble de ces paramètres est globalement déterminé par le choix des systèmes optiques insérés entre la source et l'échantillon.

Contrairement à Röntgen, l'expérimentateur bénéficie aujourd'hui d'une large panoplie de composants optiques plus ou moins sophistiqués. Toutes les optiques $\mathrm{X}$ reposent sur un des trois principes fondamentaux de l'interaction des rayons $\mathrm{X}$ avec la matière : la réflexion totale pour les miroirs, la diffraction de Bragg pour les monochromateurs, et les lentilles de Fresnel et la réfraction pour les lentilles de focalisation.

Il faut enfin souligner que les progrès réalisés dans la physique des accélérateurs font que les faisceaux de rayons $\mathrm{X}$ sont de plus en plus adaptés aux conditions expérimentales désirées. Dans ce contexte, la nouvelle tendance est de s'affranchir autant que possible de l'utilisation d'optiques, afin de limiter la dégradation du faisceau par les aberrations et la perte d'intensité liée à l'absorption ou à la diffusion.

\section{Les optiques réflexives}

Le principe physique des miroirs $\mathrm{X}$ repose sur la réflexion spéculaire : l'indice de réfraction étant inférieur à 1, la réflexion totale apparaît lorsque l'angle entre les rayons X et la surface du miroir (angle d'attaque) est suffisamment faible ; on parle alors d'incidence rasante. De manière plus quantitative, la réflexion totale n'est possible que si l'angle d'attaque est inférieur à l'angle critique $\theta_{c}$, donné par :

$$
\theta_{\mathrm{c}}=(2 \delta)^{1 / 2} \propto(\mathrm{Z} \rho / \mathrm{A})^{1 / 2} \lambda(5) .
$$

( $\mathrm{Z}$ : numéro atomique ; $\mathrm{A}$ : nombre de masse).
L'équation [5] montre la relation entre la longueur d'onde et la densité $\rho$ du matériau réfléchissant. Lorsque l'angle d'attaque est inférieur à l'angle critique, la réflectivité est théoriquement proche de $100 \%$; puis elle chute très vite audelà de l'angle critique. Cette relation impose donc l'utilisation de matériaux de haute densité tels que l'or, le platine, le rhodium ou le nickel. Même pour ces matériaux, les angles critiques sont très inférieurs au degré ; l'incidence est donc très rasante et les miroirs très longs (longueur du miroir $=$ taille du faisceau à intercepter $\times$ sinus de l'angle rasant), de l'ordre du mètre.

De plus, dans la pratique, les miroirs ne présentent jamais des surfaces idéalement polies, et la rugosité de surface atténue par diffusion diffuse l'intensité réfléchie.

Les optiques réflectives, ou miroirs $\mathrm{X}$, sont parmi les composants optiques les plus utilisés sur les lignes de lumière. Leurs fonctions sont multiples :

- Déviation ou séparation du faisceau de rayons $\mathrm{X}$.

- Filtre passe-bas lorsque, pour un angle d'incidence donné, les hautes énergies ne sont pas réfléchies alors qu'au contraire les énergies plus basses le sont. À ce titre, les miroirs $\mathrm{X}$ sont le seul moyen efficace d'absorber une partie de la charge thermique - induite par les hautes énergies - afin de protéger les autres éléments optiques en aval comme, par exemple, un monochromateur.

- Focalisation ou collimation : les miroirs $\mathrm{X}$ peuvent être courbés, soit mécaniquement, soit par polissage. La réalisation d'un miroir X elliptique (pour la focalisation d'une source ponctuelle en un point) ou parabolique (pour la collimation d'un faisceau divergent) reste cependant difficile et coûteuse, et repose sur une instrumentation très sophistiquée. L'utilisation des systèmes de focalisation basés sur les capillaires ou polycapillaires en verre existe, mais reste anecdotique.

Les nouvelles sources synchrotrons produisent un faisceau polychromatique très intense ; l'absorption d'une partie du spectre par un miroir $\mathrm{X}$, et ce quelle que soit sa fonction, fait que ce miroir est exposé à une charge thermique importante, qui peut atteindre plusieurs kilowatts en régime continu. De plus, l'utilisation de ces miroirs sous ultravide, et donc en absence de convection par l'air, complique le problème ; le gradient thermique ainsi créé est source de déformations du miroir et de dégradation de ses performances. L'ingénierie des miroirs $\mathrm{X}$ est donc une affaire complexe et repose sur des stratégies de refroidissement optimisées et de choix de matériaux appropriés.

\section{Les optiques diffractives}

Les optiques diffractives ont pour fonction première la sélection en énergie d'un faisceau de rayons $\mathrm{X}$. On parle de cristaux monochromateurs lorsqu'il s'agit de sélectionner les longueurs d'onde avant l'échantillon, et de cristaux analyseurs lorsque la sélection se fait après l'échantillon. Le principe repose sur le phénomène de diffraction, régi par la loi de Bragg (voir équation (4)). La dérivée de cette relation donne la résolution spectrale des monochromateurs cristallins, dans le cas d'un faisceau incident parallèle :

$\Delta \lambda / \lambda=\Delta E / E=\Delta \theta \cot \theta_{\mathrm{B}} \quad(6)$, où $\Delta \theta$ définit l'acceptance angulaire, appelée aussi largeur de Darwin, qui est une grandeur caractéristique d'un cristal dans une orientation et pour une énergie données. Cette largeur est très faible (inférieure à quelques microradians) pour un cristal dit parfait, et très grande (de l'ordre de plusieurs centaines de microradians) pour un cristal présentant une structure mosaïque. Les cristaux dits parfaits ont une réflectivité proche de $100 \%$; les plus utilisés sont le silicium, le germanium et le diamant. En ce qui concerne les cristaux mosaiques, ce sont le graphite et le béryllium. À partir de principes de base, une très grande variété de géométries est possible. La stratégie dépend essentiellement de la fonction du monochromateur. Par exemple : (i) le cristal peut être courbé pour focaliser le faisceau incident ; (ii) un système avec deux cristaux parallèles permet de balayer l'énergie tout en conservant la direction du faisceau émergeant fixe ; (iii) le cristal peut être taillé avec un angle par rapport au plan cristallin réfléchissant pour collimater ou élargir le faisceau ; (iv) enfin, un cristal très fin peut être utilisé en transmission dans une géométrie dite "de Laue », comme une optique séparatrice produisant deux faisceaux, l'un monochromatique et l'autre polychromatique (fig. 1c). 
Pour des valeurs de $d$ supérieures à $1,2 \mathrm{~nm}$, le choix des cristaux naturels est limité, ce qui empêche leur utilisation pour les énergies inférieures à quelques centaines d'eV (ou les longueurs d'ondes supérieures à $2,5 \mathrm{~nm}$ ). L'alternative est alors la réalisation de systèmes artificiels multicouches, qui sont constitués par des dépôts d'épaisseur contrôlée avec une alternance de couches de matériau lourd (i.e. tungstène ou platine, qui joue le rôle de milieu diffusant) et de matériau léger peu absorbant (i.e. carbone ou carbure de bore, qui assure la fonction d'espaceur). Le but visé est d'obtenir une structure lamellaire tridimensionnelle, capable de diffracter les rayons X. Les propriétés (réflectivité, résolution spectrale...) de ces multicouches sont déterminées par le choix des matériaux, le rapport des épaisseurs des matériaux lourd et léger, et le nombre de couches. L'un des principaux avantages de ces multicouches est la possibilité de faire varier ces paramètres, ce qui permet d'adapter les matériaux utilisés à de nombreuses fonctions. Par exemple, dans le cas d'optiques focalisantes très asphériques, l'angle de Bragg n'est plus constant sur la longueur de l'optique et il est alors nécessaire d'ajuster le paramètre $d$ pour satisfaire la loi de Bragg sur toute la surface de réflexion.

Pour des énergies encore plus basses, l'utilisation de réseaux de diffraction est très courante : le principe est assez similaire aux réseaux utilisés dans l'UV ou le visible, et ne sera donc pas décrit ici.

\section{Les optiques réfractives}

Comme l'avait initialement déduit Röntgen et comme le rappelle l'équation (3), la réfraction des rayons X par la matière est très faible et rend difficile la conception de lentilles similaires à celles utilisées en lumière visible. En effet, la distance focale d'une lentille mince de rayon de courbure $R$ est donnée par la relation classique définissant la focale $F$ :

$$
F=R / 2 \delta
$$

Pour les rayons $\mathrm{X}$, les petites valeurs de $\delta$ font que, même pour des petits rayons de courbure, de l'ordre de quelques centaines de microns, la distance focale est de plusieurs dizaines de mètres, ce qui est irréalisable. De plus l'indice optique étant inférieur à 1 , les lentilles de focalisation seront concaves, et non convexes comme dans le visible. Pour contourner ce problème, la stratégie est ici de créer un assemblage linéaire de $\mathrm{N}$ lentilles, dont la distance focale est alors donnée par :

$$
F=(1 / N)(R / 2 \delta)
$$

Si le matériau utilisé est faiblement absorbant (aluminium, béryllium, polymères), il est possible d'aligner plusieurs centaines de lentilles et de raccourcir la distance focale à des valeurs de quelques centimètres, ce qui rend ces lentilles utilisables dans des dispositifs expérimentaux compacts.

Un tel réseau de lentilles a été réalisé en 1996, par perçage de trous cylindriques dans un bloc d'aluminium. Depuis, les techniques de fabrication sont devenues très sophistiquées et aboutissent à deux grandes familles : les lentilles métalliques, réalisées par emboutissage, et les lentilles en silicium ou diamant CVD, réalisées grâce à des techniques de micro-lithographie.

\section{La production de sondes $X$ nanométriques}

En 1949, Paul Kirkpartick publiait un article dans Scientific American pour vanter le développement de la microscopie $\mathrm{X}$ et ses avantages sur la microscopie électronique. Les faits lui ont donné tort, et la microscopie électronique est aujourd'hui bien plus mature que son analogue avec les rayons X. En effet, il a fallu attendre les années 1990 pour assister à l'essor des instruments synchrotrons comme nouvelles sondes X.

Aujourd'hui, les développements concomitants d'optiques de rayons $\mathrm{X}$ performantes et de sources synchrotrons de petite taille, rendent possible la réalisation de sondes $\mathrm{X}$ dont les dimensions sont inférieures à $30 \mathrm{~nm}$. Comme le montre la figure 2 , les performances de focalisation des lentilles $\mathrm{X}$ sont encore meilleures ( $7 \mathrm{~nm}$ pour les $\mathrm{X}$ durs et $10 \mathrm{~nm}$ pour les $\mathrm{X}$ mous), mais la résolution spatiale des microscopes $\mathrm{X}$ est encore limitée (à environ $20 \mathrm{~nm}$ ) par de nombreux verrous technologiques comme le manque de stabilité dimensionnelle et positionnelle, et la faible résistance des échantillons aux radiations.

\section{Les systèmes de détection pour rayons $X$}

Les films photographiques sont restés très longtemps le moyen le plus courant de détection des rayons X. Aujourd'hui, à l'image de toute l'instrumentation pour rayons X, l'expérimentateur bénéficie d'une grande palette de solutions adaptées à ses besoins. En particulier, les développements réalisés en astrophysique et en imagerie médicale ont abouti à des progrès technologiques qui ont des retombées positives sur l'instrumentation synchrotron.

Globalement, les détecteurs de rayons $\mathrm{X}$ peuvent être classés en trois grandes catégories, selon le type d'interaction physique entre leurs constituants et les photons : les détecteurs à scintillation, les détecteurs à gaz et les détecteurs solides.

\>
2. Évolution des dimensions des faisceaux de rayons $X$ dans le plan focal d'une lentille pour la production de sondes $X$, pour les rayons $X$ mous $(<2 \mathrm{keV})$ et tendres/durs ( $>2 \mathrm{keV})$.

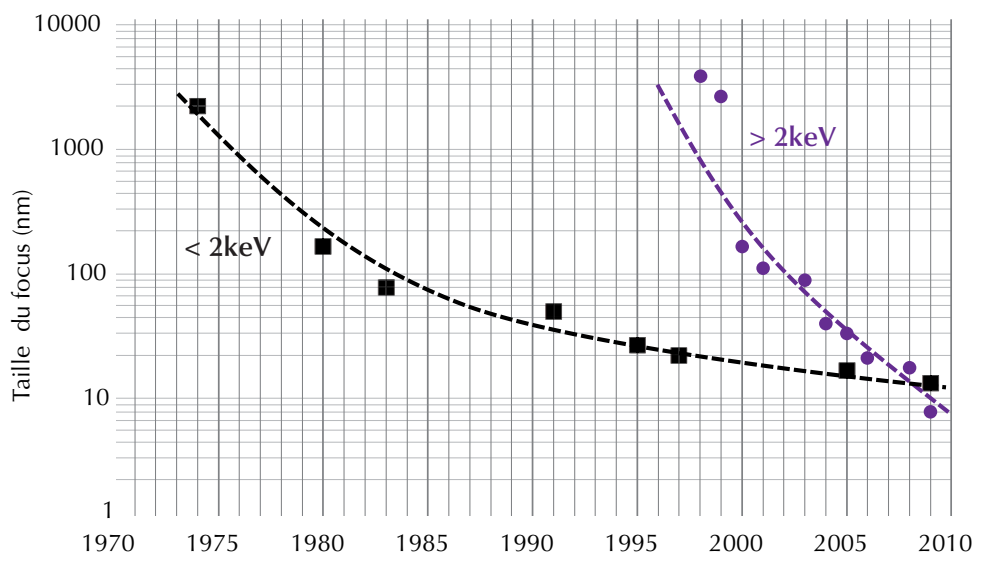

Année 
>>

Les détecteurs unidimensionnels

Les scintillateurs sont très répandus et reposent sur la conversion des photons $\mathrm{X}$ en photons visibles au sein d'un cristal dopé (i.e. NaI dopé au thallium); le signal ainsi produit est traité et amplifié par un photomultiplicateur.

Les compteurs à gaz sont des détecteurs dans lesquels les rayons $\mathrm{X}$ ionisent un gaz rare. Les électrons ainsi produits sont ensuite collectés sur une anode. La tension appliquée détermine le mode d'amplification du phénomène d'ionisation, critère qui définit le type de détecteur : chambre à ionisation, compteur proportionnel ou compteur Geiger-Muller. Les détecteurs à gaz peuvent être plus sophistiqués, avec plusieurs anodes, et offrir une résolution spatiale - donnée par le temps pour un électron créé d'atteindre l'anode.

Enfin, les détecteurs semi-conducteurs sont le produit des technologies microélectroniques à base de silicium ou de germanium. Leur bonne résolution en énergie - de l'ordre de $130 \mathrm{eV}$ pour les meilleurs font que ces détecteurs sont très utilisés pour l'analyse élémentaire de la fluorescence X. Très répandus sur les sondes électroniques de laboratoire (EDX), ces détecteurs sont également utilisés sur les lignes de lumière synchrotron, mais dans des configurations plus sophistiquées. Par exemple, plusieurs détecteurs sont accolés dans des géométries complexes permettant d'optimiser l'angle solide de détection.
Depuis quelques années, un nouveau type de détecteur - dit SDD pour Silicon Drift Diode - connait un grand succès pour ce type d'applications, car il surpasse ses concurrents en termes de taux de comptage.

\section{Les détecteurs bidimensionnels}

Les détecteurs bidimensionnels (2D) permettent l'enregistrement d'une image et sont en pratique une extension des films à rayons $\mathrm{X}$ tels que ceux utilisés par Röntgen il y a 150 ans, ou encore aujourd'hui pour certaines applications de radiographie médicale. La possibilité d'enregistrer des images numérisées a véritablement révolutionné toutes les disciplines utilisant le rayonnement synchrotron. La diffraction et la diffusion, les techniques d'imagerie et certaines spectroscopies exploitent les nouvelles possibilités offertes par les détecteurs bidimensionnels pour rayons $\mathrm{X}$.

Le capteur le plus utilisé est le CCD - Charge Coupled Device - très semblable aux capteurs utilisés dans les caméscopes numériques. Un CCD est un capteur sensible à la lumière visible, et consiste en une matrice de pixels imprimée sur une plaque de silicium. Les photons absorbés dans le silicium génèrent des électrons qui sont alors piégés dans chaque pixel, et l'enregistrement de l'image se fait ligne par ligne. Plus récemment, suivant la même évolution que les caméscopes commerciaux, les capteurs CMOS - Complementary Metal
Oxide Semiconductor - sont également intégrés dans les détecteurs 2D pour rayons $\mathrm{X}$.

Les détecteurs 2D peuvent être scindés en deux classes, avec des systèmes de détection directe ou indirecte. La détection directe, où le capteur est directement illuminé par les rayons $\mathrm{X}$, est plus simple et permet de s'affranchir des dégradations ou des pertes générées par les éléments optiques utilisés dans la détection indirecte. De plus, associée à une électronique très sophistiquée, la détection directe permet le comptage précis du nombre de photons par pixel, avec un bruit électronique proche de zéro. En revanche, la plupart des capteurs CCD existant sur le marché sont optimisés pour la détection de lumière visible, et ont de ce fait une faible efficacité dans la gamme des rayons $\mathrm{X}$. L'alternative la plus commune est alors la détection indirecte, dans laquelle un convertisseur de rayons $\mathrm{X}$ en lumière visible associé à un système optique est inséré devant le capteur (fig. 3). La résolution spatiale peut être déterminée par la taille du pixel lorsque le capteur est directement illuminé par les rayons X. Dans de nombreuses applications, un convertisseur de rayons $\mathrm{X}$ en lumière visible, associé à un système optique, est inséré devant le capteur. Dans cette configuration, la résolution peut être améliorée grâce au facteur d'agrandissement du système optique. Des résolutions inférieures à $0,5 \mu \mathrm{m}$ sont courantes.
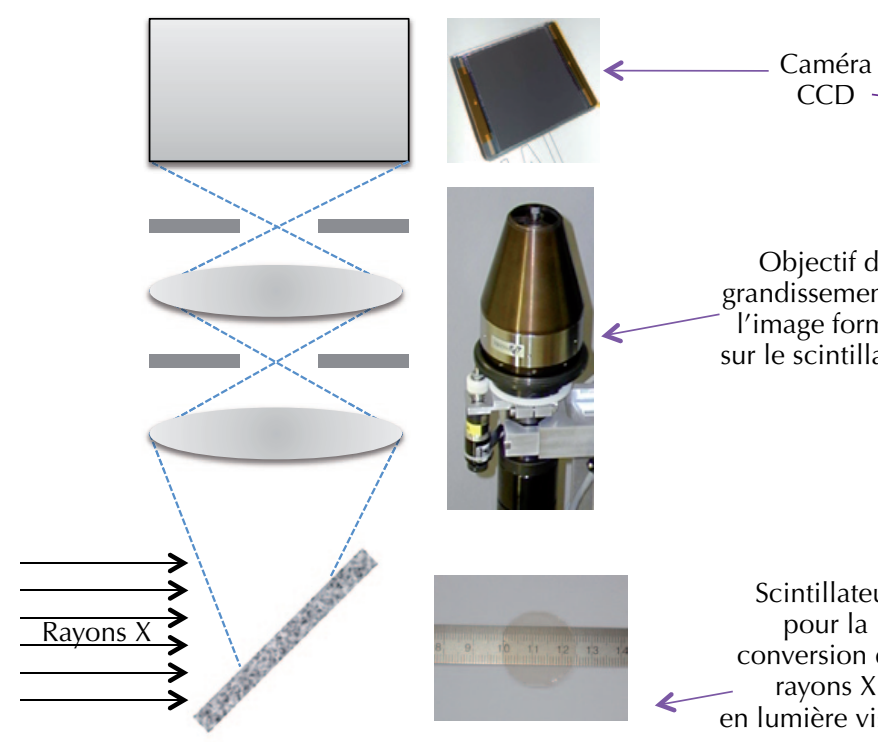

Objectif de grandissement de I'image formée sur le scintillateur 
Par ailleurs, les prises d'images sont de plus en plus rapides, comme illustré sur la figure 4. Il est aujourd'hui possible d'enregistrer plusieurs milliers d'images en une seconde, ce qui correspond à un volume de données numériques de plus en plus difficile à gérer. À titre d'exemple, l'ensemble des expériences de l'ESRF produit plus de 3 téraoctets (3000 Gb) par jour, et il est prévu que ce chiffre soit multiplié par 100 dans les années à venir. Les problèmes de stockage et de transfert de ces données, mais aussi les moyens de calcul associés pour manipuler ces gros volumes de données deviennent très critiques, malgré les progrès des outils informatiques.

\section{L'instrumentation autour de l'échantillon}

L'un des atouts principaux des rayons $\mathrm{X}$ est leur pouvoir de pénétration des matériaux et, de ce fait, la possibilité de réaliser des mesures dans des conditions in situ ou in operando contrôlées. Il est donc logique que la communauté scientifique utilisant le rayonnement synchrotron ait beaucoup investi dans la réalisation d'environnements autour de l'échantillon, spécifiques aux problématiques étudiées. Il serait trop ambitieux de présenter ici un panorama exhaustif de ces développements. Nous nous limitons donc à résumer les grandes tendances pour trois domaines scientifiques majeurs.
- La biologie structurale : la détermination de la structure tridimensionnelle de protéines, basée sur la diffraction $\mathrm{X}$ à partir de cristaux synthétisés, a motivé un très grand nombre de développements visant en particulier à pousser le rendement de mesures. Ainsi, le haut degré d'automatisation des expériences a permis de minimiser l'intervention humaine et de maximiser la production de mesures à un niveau très similaire à ce que l'on trouve dans le monde industriel. Les échantillons sont positionnés dans l'appareil de mesure par des robots, le cristal est automatiquement aligné sur le faisceau de rayons $\mathrm{X}$ grâce à des algorithmes de reconnaissance de forme, et le temps de mesure est réduit à son minimum pour limiter les dommages induits par les radiations (voir l'article de M. Bowler et D. Nurizzo, p. 48). Les projets les plus aboutis dans ce domaine visent à mesurer plus de 2000 cristaux par jour.

- La science des matériaux en conditions extrêmes : il s'agit, par excellence, du domaine scientifique où l'environnement des échantillons est d'une importance fondamentale et souvent la clé du succès. Il couvre de nombreux champs d'applications tels que la synthèse de nouveaux matériaux, l'étude physicochimique des géosystèmes, et la validation de théories ab initio. Pour les études statiques, les cellules à enclume diamant soumettent de faibles volumes d'échantillons (quelques millimètres cubes) à des pressions de $200 \mathrm{GPa}$ ( 2 millions de bars) et des températures proches de $5000 \mathrm{~K}$, en y associant un chauffage par laser de puissance (voir l'article de M. Mezouar et al., p. 70).

- Les études de surface sont, par nature, un domaine de recherche nécessitant un environnement très contrôlé. En particulier, des développements instrumentaux remarquables ont été réalisés pour étudier à l'aide des rayons $\mathrm{X}$ des surfaces intégrant des nanostructures (voir l'article de G. Renaud, p. 65). Ces études sont en général effectuées in situ ou in operando, depuis les conditions de l'ultravide $\left(10^{-14}\right.$ bar) jusqu'à celles de la catalyse (sous typiquement un mbar à un bar de gaz réactifs), en passant par celles de l'élaboration de ces structures par voies physiques (évaporation, ablation laser) ou chimiques, en contrôlant précisément les pressions partielles et les températures. Une tendance actuelle est de miniaturiser ces environnements complexes, afin d'étudier des nano-objets uniques en conditions réelles.

(a) Les gammes de longueurs d'onde couvertes par SOLEIL et l'ESRF sont montrées dans la figure 3 de l'article de M.E. Couprie et al., voir p. 22.

(b) W.H. et W.L. Bragg, père et fils, ont reçu le prix Nobel en 1915 "pour leurs travaux d'analyse des structures cristallines à l'aide des rayons X ".

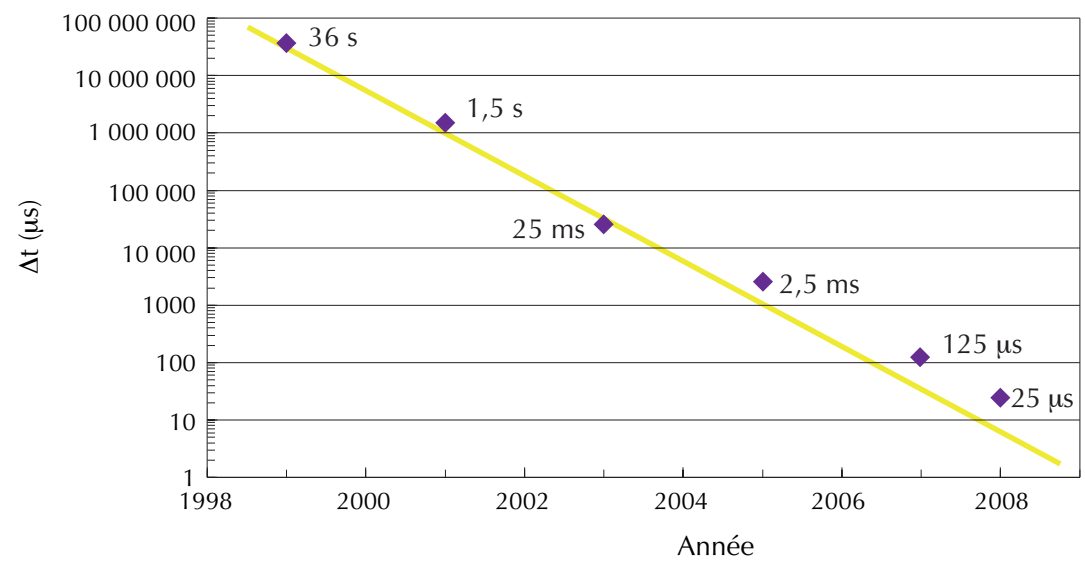

4. Évolution des temps d'obtention d'une image. En 10 ans, le développement de nouveaux détecteurs bidimensionnels a permis de passer de l'enregistrement de 100 images en 1 heure à 100 images en 2 ms.

\section{En savoir plus}

- D. Attwood, Soft X-Rays and Extreme Ultraviolet Radiation: Principles and Applications, Cambridge University Press (2000)

- P. Goudeau et R. Guinebretière, Rayons X et matière - RX 2009, Hermès - Lavoisier (2010).

- C. Esnouf, Caractérisation microstructurale des matériaux - Analyse par les rayonnements X et électroniques, Métis Lyon Tech, Presses polytechniques et universitaires romandes (2011).

- J. Doucet et J. Baruchel, « Rayonnement synchrotron et applications ", Les Techniques de l'Ingénieur, P2700 (2011). 